MATEC Web of Conferences 44, 02086 (2016)

DOI: $10.1051 /$ matecconf $/ 20164402086$

(C) Owned by the authors, published by EDP Sciences, 2016

\title{
Design and simulation of a novel circularly polarized antenna with polarization reconfigurable characteristics
}

\author{
Hai ZHANG ${ }^{a}$, Wei Guo ZHANG, Yue He GE and Shan Ce LV \\ College of Information Science and Engineering, Huaqiao University, Xiamen 361021, China
}

\begin{abstract}
A novel circularly polarized antenna with polarization reconfigurable characteristics was designed using co-simulation of Ansoft HFSS and Designer software. It consists of a dual-polarized antenna and phase switching network which act as the feed network for the dual-polarized antenna. The phase switching network was designed based on a Wilkinson power divider, where the output port was connected with SPDT to form a switching network. By controlling the SPDT state-off / on, the phase difference of the two ports could be alternated, which generated the orthogonal modes between the two ports of dual-polarized antenna. So that Left-hand circular polarization (LHCP) and Right-hand circular polarization (RHCP) could be achieved. The simulation shown that reflection coefficient was less than $-12 \mathrm{~dB}$ and the axial ratio was below $3 \mathrm{~dB}$ between $1.8 \mathrm{GHz}$ and $2.4 \mathrm{GHz}$ with polarization reconfigurable characteristics.
\end{abstract}

\section{Introduction}

Re-configurable antenna has a lot of advantages. It has been widely used in both military and civilian fields. Reconfigurable antenna can be divided to frequency reconfigurable antennas, pattern re-configurable antennas, polarized re-configurable antennas and now hybrid reconfigurable antennas have been developed [1-3]. Polarized re-configurable antennas have anti-multipath fading effect and have the advantages of improving frequency reuse[4,5]. This reference uses slot structure and uses the PIN as a switch to control the current flow path and become the orthogonal polarization modes[6]. But the band of 3DB axial ratio is relatively narrow and opening slots will have impact on the stability of entire antenna. This reference uses MEMS as a switch and toggle polarization by controlling two corners of rectangular micro-strip antenna[7-9]. However, the reaction time of MEMS is very slow and it is unsuitable for high speed data communication applications. Besides, traditional re-configurable antennas use diode as a switch and they require sophisticated complex of DC bias circuit. Based on the dual-polarized antenna, the paper shows a novel circularly polarized antenna with polarization reconfigurable characteristics[10,11]. It consists of a dualpolarized antenna and phase switching network which act as the feed network for the dual-polarized antenna. The phase switching network was designed based on a power divider, where the output port was connected with SPDT to form a switching network. A novel circularly polarized antenna with switched polarization direction can be made in this method.

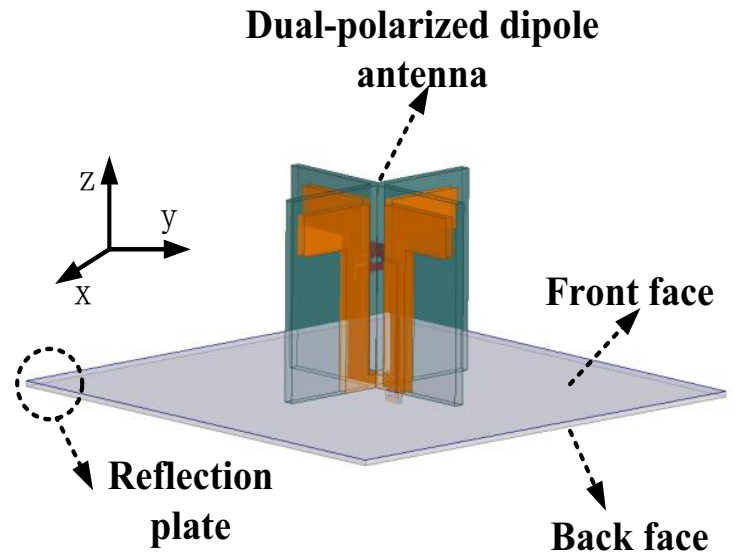

Figure 1. Structure of polarized re-configurable antenna.

\section{Operating principle}

Dual-polarized antenna including vertical polarization and horizontal polarization, which have two orthogonal field components, when the two orthogonal field components obey formula (1), the polarization of the radiation field is a circular polarization. The polarization will be left-hand circular polarization (LHCP) if $\sigma=\pi / 2$ while it will be right-hand circular polarization (RHCP) if $\sigma=-\pi / 2$, under the condition of $\left|E_{1}\right|=\left|E_{2}\right|$.

$$
\vec{E}=E_{x} \vec{x}+E_{y} \vec{y}=E_{1} \cos \varpi t \vec{x}+E_{2} \cos (\varpi t+\sigma) \vec{y}
$$

So we can change the polarization between LHCP and RHCP by controlling the difference between the phase polarization.

\footnotetext{
${ }^{\mathrm{a}}$ Author to whom correspondence should be addressed. Electronic mail: xjtuzhanghai@163.com.
} 


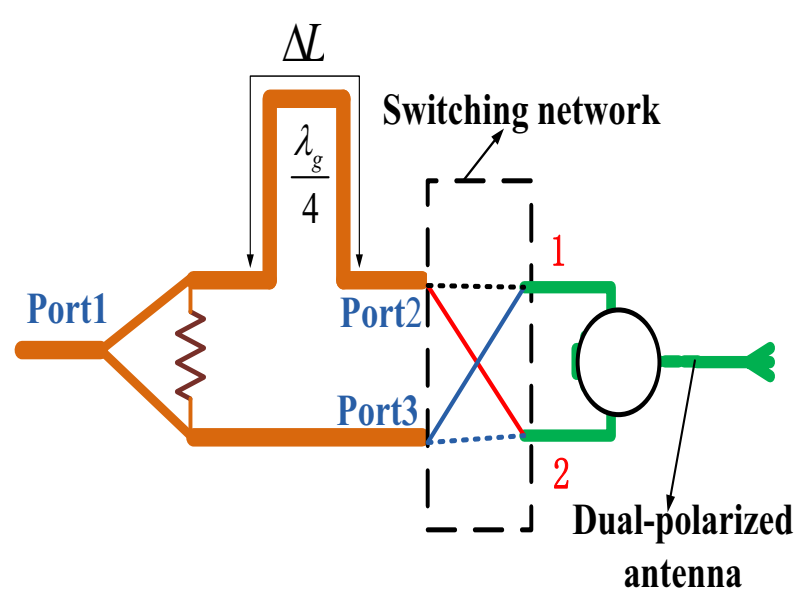

Figure 2. Principle diagram of feeding network.

The circular polarization reconfigurable antenna designed in this article is shown in figure 1, which is consisted by a dual-polarized antenna and a phase switching network. The dual-polarized antenna is consisted by a dualpolarized dipole antenna and a reflector, the function of phase switching network is to make the signal amplitude of two ports of the antenna equal and let the parameter $\sigma$ switch between positive and negative polarity capable. The phase switching network using micro-strip structures and was designed in the back of the reflector so that it has the same shape with the antenna.

According to the analysis above, the phase switching network is shown in figure2, due to the micro-strip structure of the feed network, the electromagnetic wave in this antenna is Quasi-TEM mode[12-14]. So the electric field is orthogonal when radiated by the dualpolarization antenna. we use a bisection Wilkinson divider as the power network,port2 and port3 are connected with the two feed ports by the switching network, and port 2 is $\lambda_{g} / 4$ longer than port 3 , that is $\beta \times \Delta L=\pi / 2$ degree different, which means the phase of port2 is $\pi / 2$ degree slower than that of port3. When switch the network, turn on the solid line and turn off the dotted line, the signal amplitude of port 1 and port 2 of the antenna is equal while the phase of signal from port1 is $\pi / 2$ degree faster than that of port 2 , the radiation field is left-hand circular polarized.

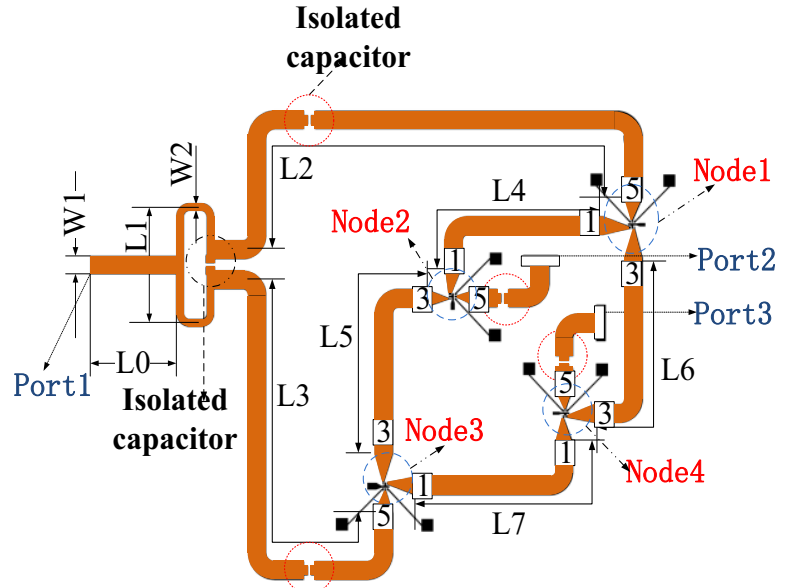

Figure 3. Structure of feeding network.
Otherwise, we will get a right-hand circular polarization field. That means we can change the polarization of the antenna by controlling the working state of the switching network.

\section{Simulation Study}

The dual-polarized antenna designed of cross type of printed dipole is shown in figure3, the material of the substrate is FR4, and the parameters are $\varepsilon_{r}=4.4$, $h=1.6 \mathrm{~mm}$. The specific principle and procedure can be referred to document [15-17]. The main introduction here is about the design of the phase switching network. According to the theory above, the phase switching network is as what as shown in figure 3 , to make the network the same shape of the antenna, the locations of port 2 and port 3 are designed steady. In order to prevent the RF ports from been destroyed by the overload DC voltage, a capacitance is used in the antenna. The node in figure3 is the location of switch SPDT, the function of the switch is to turn the circuit on or turn it off. The dimensions of the phase switching network L2-L7 are as follows[18-20]:

$$
\begin{gathered}
L_{5}=L_{7}, L_{4}=L_{6} \\
\Delta L=\left(L_{2}+L_{4}\right)-\left(L_{3}+L_{5}\right)=\lambda_{g} / 4 \\
\beta \times \Delta L=\pi / 2
\end{gathered}
$$

Setting the VCTL1 $=0$, VCTL2 $=1$ (control site of SPDT ) to be the first control state, the VCTL1=1, VCTL2 $=0$ to be the second control state.

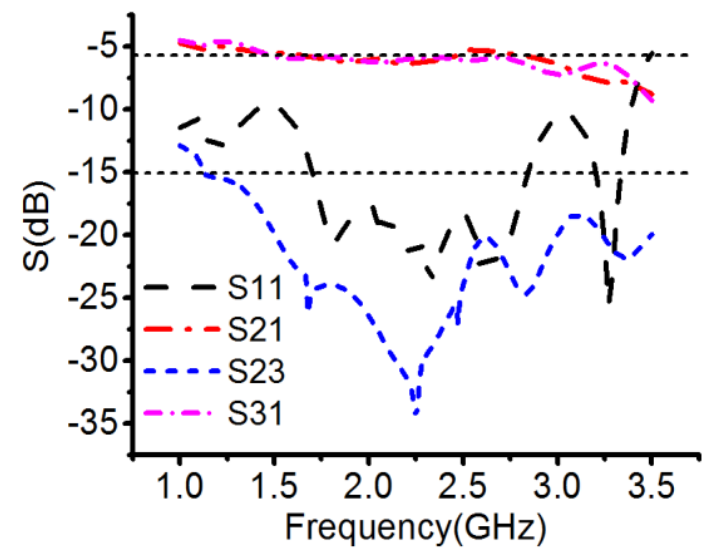

Figure 4. Simulation result of $\mathrm{S}$ parameter in state 1

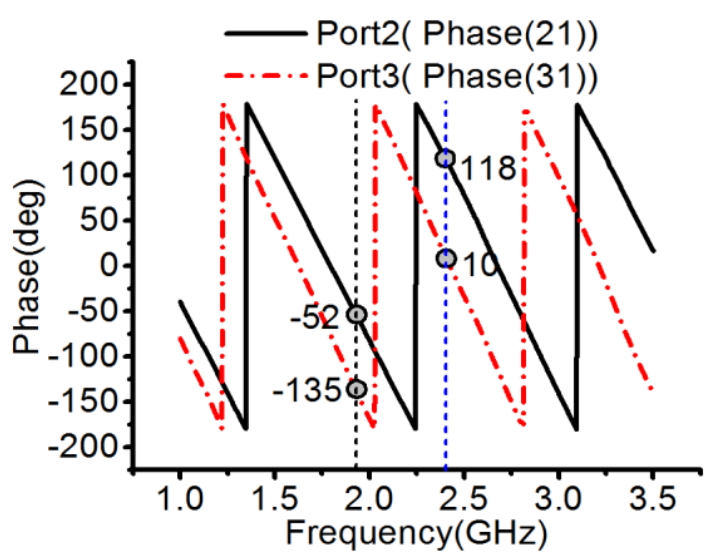

Figure 5. Simulation result of phase in state 1 


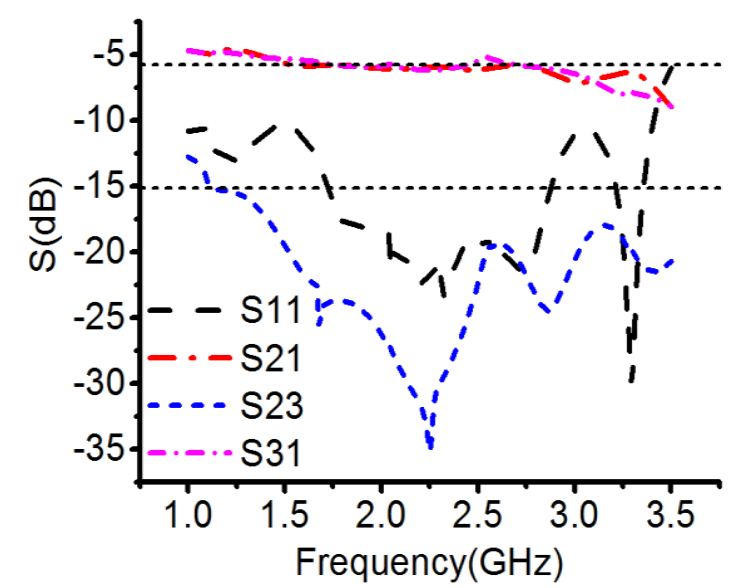

Figure 6. Simulation result of $\mathrm{S}$ parameter in state 2

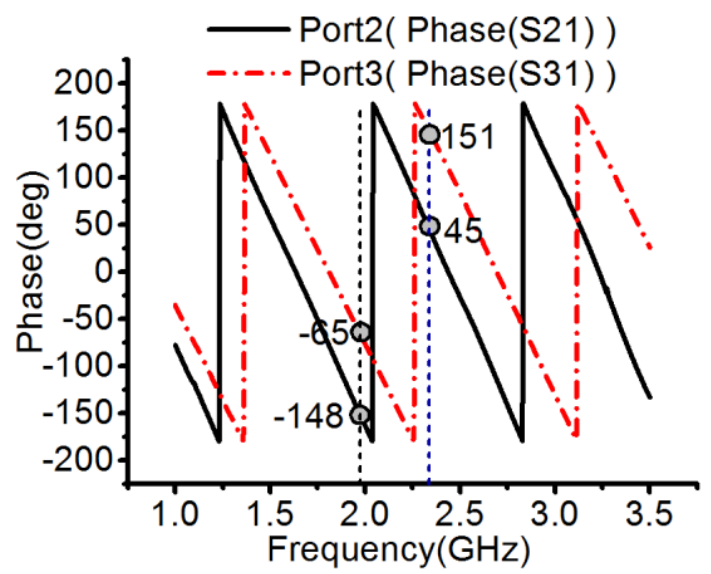

Figure 7. Simulation result of phase in state 2

when on the first control state, the node of 1 and 5 is connected and the node of 3 and 5 is cutting off. The routes of the two signals from power divider to the port 2 and port 3 are $\mathrm{L} 2 \rightarrow \mathrm{L} 4 \rightarrow$ port 2 and $\mathrm{L} 3 \rightarrow \mathrm{L} 7 \rightarrow$ port3. So the phase of port 2 lags to port 3 about $\pi / 2$. When on the second control state, the node of 3 and 5 is connected and the node of 1 and 5 is cutting off. The routes of the two signals from power divider to the port 2 and port 3 are $\mathrm{L} 2 \rightarrow \mathrm{L} 6 \rightarrow$ port3 and L3 $\rightarrow \mathrm{L} 5 \rightarrow$ port2. So the phase of port 2 runs ahead of port 3 about $\pi / 2$. Because of the equal power division network, the amplitude of the two output ports in the two control states are equality[21].

According to the above analysis, accomplishing the simulation of the feed network in the Ansoft Designer. Adjusting the length of L2、L3 to make the phase of port 2 and port 3 differ $\pi / 2$ when centre frequency is 2.25 GHz. In the simulation, I have used the $\mathrm{S}$ parameter from the SPDT manufacturer and considered the actual packaging of all components. The result of the simulation is shown in the figures 4 to 7 .

According to the simulation, when the phase switch network is in the $1.8 \sim 2.4 \mathrm{GHz}$, the insertion loss $\mathrm{S} 21$ 、 S31 of port 2 and port 3 are both about $-6 \mathrm{~dB}$, the reflection coefficient $\mathrm{S} 11$ is less than $-15 \mathrm{~dB}$, the isolation S23 is less than $-20 \mathrm{~dB}$, the skewing of phase is $90^{\circ} \pm 10^{\circ}$.In addition, when on the first control state, the phase of port 2 lags to port 3 about $\pi / 2$. When on the second control state, the phase of port 2 runs ahead of port 3 about $\pi / 2$.So we can switch the phase by controlling the state of SPDT. By using dynamic united simulation function of the HFSS and Ansoft Designer to accomplished the united simulation of dual polarized antenna and phase switching network. The result of the simulation is shown by figures $8-11$. Finally, according to the result of united simulation which in the frequency band from $1.8 \mathrm{GHz}$ to $2.4 \mathrm{GHz}$, the antenna work in the left-hand circular polarization and right-hand circular polarization under the two control states, and the axial ratio of it is less than $3 \mathrm{~dB}$.

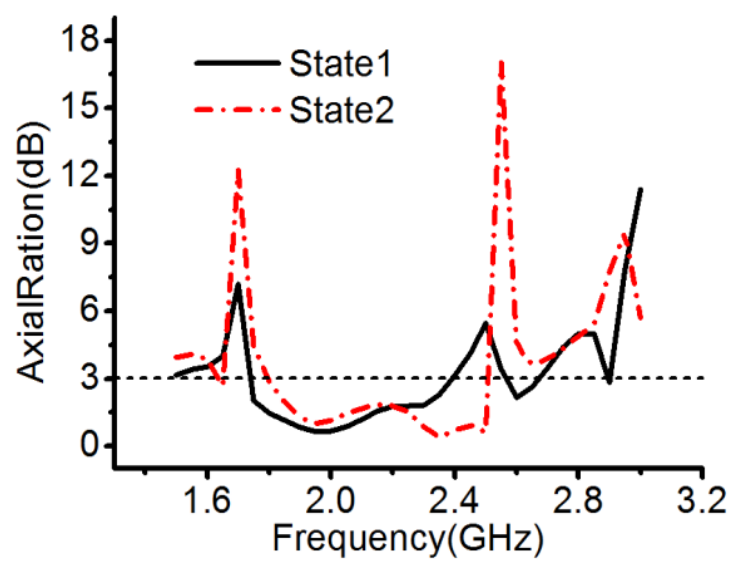

Figure 8. Simulation result of AR in state 1,2

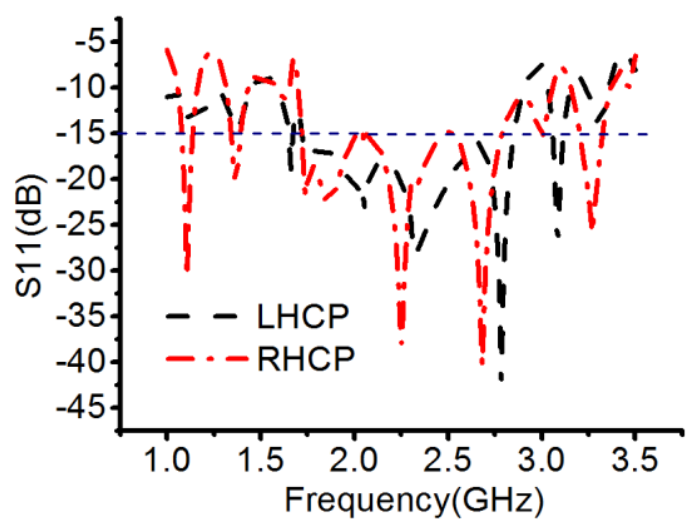

Figure 9. Simulation result of reflection coefficient in state 1,2

\section{Conclusions}

This paper combines the SPDT with a Wilkinson power divider to build a novel phase switching network. The switching network acts as the feed network for circularly polarized antenna. By controlling the levels between port VCTL1 and port VCTL2 of SPDT, left/right hand circular polarization direction can be switched fast. The co-simulation of Ansoft HFSS and Designer software shown that reflection coefficient was less than $-12 \mathrm{~dB}$ and the axial ratio below $3 \mathrm{~dB}$ between $1.8 \mathrm{GHz}$ and $2.4 \mathrm{GHz}$ with polarization re-configurable characteristics. All the results shown that the performance of proposed circularly polarized antenna is fine. 


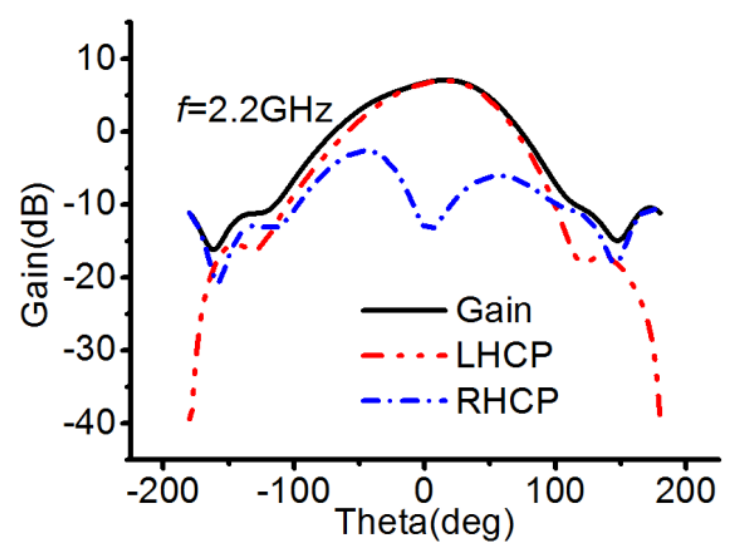

Figure 10. Simulation result of gain in state 1

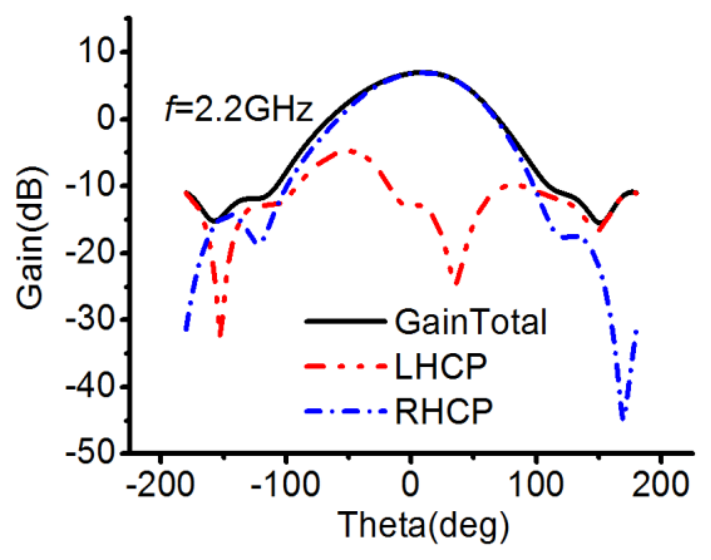

Figure 11. Simulation result of gain in state 2

\section{Acknowledgments}

This work was financially supported by the Project of Education Department of Fujian Province, China (Grant No. JA14022), and the Undergraduate Education \& Teaching Reform Project of Huaqiao University, China (Grant No.2013JGYB36), Xiamen, Fujian province, China. The authors also want to thank the professor Ge and professor Tang for their kindly discussions.

\section{References}

1. Tian Yubo, Tian Guannan. Overview of researches on reconfigureabe antennas, Journal of Jiangsu University of Science and Technology, 2012, 03:271-275.

2. Z-Y Zhang, N-W Liu, J-Y Zhao, Wideband circularly polarized antenna with gain improvement, Antennas and Wireless Propagation Letters, Vol.12, 2013,pp:456-459.

3. M-J Li, K. M. Luk, A wideband dual-fed circularly polarized antenna, Electromagnetics, 2003,pp:112-114.

4. Fa-Shian Chang, Kin-Lu Wong, Tzung-Wern Chiou. Low-Cost Broadband Circularly Polarized Patch Antenna. IEEE Transactions on Antennas And Propagation, 2003, 51(10):3006-3009.

5. Y-J Hu, Z-M Qiu, B Yang, S-J,Shi, Design of novel wideband circularly polarized antenna based on Vivaldi Antenna structure, Antennas and Wireless Propagation Letters,2015,pp.1.
6. Fan Yang, Yahya Rahmat-Samii. A Reconfigurable Patch Antenna Using Switchable Slots for Circular Polarization Diversity[J]. IEEE Microwave And Wireless Components Letters, 2002, 12(3):96-98.

7. Shih-Hsun Hsu, Kai Chang. A Novel Reconfigurable Microstrip Antenna with Switchable Circular Polarization. IEEE Antennas And Wireless Propagation Letters, 2007,6:160-162.

8. E-C Wang, Y-C Liu, S-J,Fang, P, Cheng, Wideband circularly polarized antenna for GPS/Glonass,2010, pp.2511-2513.

9. L Chen, T-L Zhang, C Wang, X-W Shi, Wideband circularly polarized microstrip antenna with wide beamwidth, Antennas and Wireless Propagation Letters, Vol.13,2014,pp:1577-1590.

10. Jeen-Sheen Row, Chuang-Jiashih Shih. PolarizationDiversity Ring Slot Antenna With Frequency Agility. IEEE Transactions On Antennas And Propagation, 2012, 60(8):3953-3957.

11. Hu Yue,Yang Xuexiao,Lu Zhongliang,and Zheng Guoxin. A novel polarized reconfigurable microstrip, Journal of microwave, 2014, 24 (3): 5-8.

12. Wang Kewei, Wang Junhong. Study on the transmission and reflection properties of the microstrip line bend, Journal of microwave, 2006, 22(3):32-35.

13. A. Khalajmehrabadi, M. K. A. Rahim, and N. A. Murad: Planar Wideband Circularly Polarized Dielectric Resonator Antenna, European Conference on Antennas and Propagation (EUCAP) (2012).

14. W. Liu, K. Wei, Z. Zhang, and J. Zheng: A circularly polarized antenna with conical beam, IEEE EDAPS (2004).

15. Wang Can, Ge Yuehe. A novel design of broadband dual-polarized printed dipole antenna for base stations, Journal of Huaqiao University, 2014, 35(2):132-135.

16. C.Wang, Y.H. Ge: Journal of Huaqiao university (Natural science), Vol.35,2014, pp.132-135.

17. C.Wang, Y.H. Ge: A Novel Broadband Printed Dipole Antenna and Its Application for TD-LTE Communications, International Journal of Antennas and Propagation(2014).

18. Wang Anguo, Dong Jiawei, and Wang Peng. Design of printed dipole antenna with reconfiguration radiation pattern, Journal of Radio Science, 2010, 25(1):190-193.

19. Mak A C K, Rowell C R, Murch R D. Low cost reconfiguration land restorer planar antenna array, IEEE transactions on antennas and propagation, 2009, 57(10): 3051-3061.

20. Raj.R, Poussot B, Laheurte J M, et al. Pattern reconfiguration of micro strip antenna using flip-clip mounted package MEMS, Microwave and Optical Technology Letters, 2010,52(3):574-577.

21. Bai Yangying, Wang Bingzhong, et al. Applying weighted thinned linear array and pattern re-configurable elements to extend pattern scanning range of millimeter wave microstrip phase array, Journal of Inferred, Millimeter, and Terahertz Waves,2010,31(1):1-6. 